\title{
An analysis of multiple superheated and saturated regions in a stagnant binary film
}

\author{
H. J. H. Brouwers ${ }^{\dagger}$
}

Akzo Research Laboratories Arnhem. Fibers Division, Department of Mechanical Engineering, Velperweg 76, 6824 BM Arnhem. The Netherlands

(Received 19 April 1991 and in final form 19 July 1991)

\section{INTRODUCTION}

IN THE binary film analyses of Brouwers [1, 2], the presence and the magnitude of a saturated region in the film were determined. For both superheated and saturated regions energy and diffusion equations were derived and solved. A major assumption of these analyses was that only one superheated and one saturated region were allowed to occur. which was not proved for $L e_{v}<1$. Accordingly, in this technical note the fog film model is first recapitulated. Subsequently, it will be examined whether multiple superheated and saturated regions in the film are possible and if the aforementioned assumption is correct.

\section{THE FILM WITH A SINGLE SATURATED REGION}

A film is considered in which heat and diffusional mass transfer occur from bulk to wall, $c_{b}>c_{i}$ and $t_{n}>t_{i}$, as found in a condenser. When superheat in the entire film is assumed, the temperature and vapour mass fraction profiles follow from the energy and diffusion equation using as boundary conditions $t\left(y=\delta_{\mathrm{t}}\right)=t_{\mathrm{b}}, c\left(y=\delta_{\mathrm{i}}\right)=c_{\mathrm{h}}, t(y=0)=t_{\mathrm{i}}$ and $c(y=0)=c_{\mathrm{i}}$ (sec Fig. 1). Lliminating $y$ from the resulting $t(y)$ and $c(y)$ yields the following relation between $c$ and $t$ in the superheated film [1]:

$$
\begin{aligned}
& \delta_{\mathrm{c}}<\delta_{\mathrm{t}}\left[\begin{array}{rl}
c^{\prime}=G(t)= & 1+\left(c_{\mathrm{i}}-1\right)\left(\frac{t-t_{\mathrm{i}}}{t_{\mathrm{b}}-t_{\mathrm{i}}}\right. \\
& \left.\times\left(\exp \left(\frac{\delta_{\mathrm{i}}}{L e_{\mathrm{i}} \delta_{\mathrm{c}}} \ln \left(\frac{1-c_{\mathrm{b}}}{1-c_{\mathrm{i}}}\right)\right)-1\right)+1\right)^{\prime \prime}
\end{array}\right. \\
& \left(t_{i} \leqslant t \leqslant t\left(y=\delta_{i}\right)\right)
\end{aligned}
$$$$
\delta_{c}=\delta_{1}: c=G(t)=1
$$$$
+\left(c_{i}-1\right)\left(\frac{t-t_{i}}{t_{h}-t_{i}}\left(\exp \left(\frac{1}{L e_{v}} \ln \left(\frac{1-c_{n}}{1-c_{i}}\right)\right)-1\right)+1\right)^{L e_{v}}
$$

$$
\delta_{c}>\delta_{1}\left[\begin{array}{rl}
c-G(t)- & 1+\left(c_{\mathrm{i}}-1\right)\left(\frac{t-t_{\mathrm{i}}}{t_{\mathrm{b}}-t_{\mathrm{i}}}\right. \\
& \left.\times\left(\exp \left(\frac{\delta_{\mathrm{t}}}{L e_{\mathrm{v}} \delta_{\mathrm{c}}} \ln \left(\frac{1-c_{\mathrm{b}}}{1-c_{\mathrm{i}}}\right)\right)-1\right)+1\right)^{L_{c}} \\
& \left(t_{\mathrm{i}} \leqslant t \leqslant t_{\mathrm{b}}\right)
\end{array}\right.
$$

$\dagger$ Present address: Department of Civil Engineering, Twente University, P.O. Box 217, 7500 AE Enschede, The Netherlands.

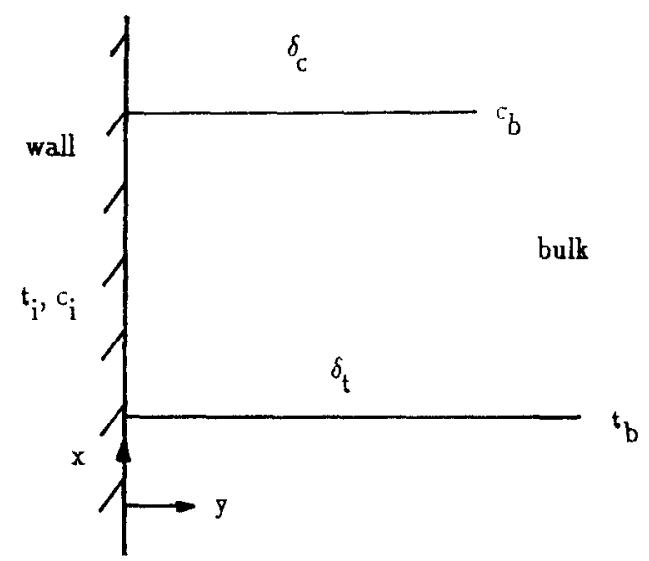

Fig. 1. The stagnant film.

This monotonically increasing function in $t$ is concave for $L e_{\mathrm{v}}>1$, convex for $L e_{\mathrm{v}}<1$ and straight for $L e_{\mathrm{v}}=1$ [ [1]. For mass transfer from bulk to wall. possible supersaturation in the film is examined with the following slope condition $[1,2]$ :

$$
\left.\frac{\mathrm{d} F}{\mathrm{~d} t}\right|_{t_{1}}<\left.\frac{\mathrm{d} G}{\mathrm{~d} t}\right|_{,}-L \mathcal{e}_{\mathrm{v}} \frac{c_{\mathrm{i}}-1}{t_{\mathrm{h}}-t_{\mathrm{j}}}\left(\exp \left(\frac{\delta_{\mathrm{t}}}{L e_{\mathrm{b}} \delta_{\mathrm{c}}} \ln \left(\begin{array}{c}
1-c_{\mathrm{b}} \\
1-c_{\mathrm{i}}
\end{array}\right)\right)-1\right)
$$

In case equation (1) is not satisfied, the curve $G(t)$ is situated entirely in the superheated region, and fog will not be formed. On the other hand, if this requirement is satisfied. the curve $G(t)$ intersects the saturation line $F(t)$. Assuming no supersaturation to be possible, fog will then be formed in part or in the entire film.

The next step is then to distinguish a superheated and saturated region in the film. The temperature and vapour mass fraction at the boundary of both regions $\left(\right.$ at $y=\dot{\delta}_{i i}$ ) are referred to as $t_{i t}$ and $c_{i d}$, respectively (see Fig. 2).

The vapour mass fraction in the saturated region up to and including the boundary with the superheated region is related to the temperature by the saturation condition

$$
c=F(t) \quad\left(t_{i} \leqslant t \leqslant t_{i}\right) .
$$

In the superheated region $c$ and $t$ are again related by equation (1), but $t_{i}$ and $c_{i}$ have now been replaced by $t_{i 1}$ and $c_{i}$. respectively

$$
\begin{aligned}
c & =G(t) \\
& =1+\left(c_{\mathrm{at}}-1\right)\left(\frac{t-t_{\mathrm{a}}}{t_{\mathrm{b}}-t_{\mathrm{a}}}\left(\exp \left(\frac{1}{L e_{\mathrm{b}}} \ln \left(\frac{1-c_{\mathrm{b}}}{1-c_{\mathrm{b}}}\right)\right)-1\right)+1\right)
\end{aligned}
$$

$$
\left(t_{\mathrm{i} 1} \leqslant t \leqslant t_{\mathrm{b}}\right) \text {. }
$$

In deriving this equation it has been assumed that the diffusional and thermal film thicknesses are equal, thus $\delta_{i}=\delta_{1}$. This assumption has no consequences for the investigation 


\section{NOMENCLATURE}

\begin{tabular}{|c|c|}
\hline$c$ & vapour mass fraction \\
\hline $\mathbb{D}$ & diffusion coefficient $\left[\mathrm{m}^{2} \mathrm{~s}^{-1}\right]$ \\
\hline$F$ & saturation vapour mass fraction \\
\hline$G$ & $\begin{array}{l}\text { relation between } c \text { and } t \text { in superheated } \\
\text { region }\end{array}$ \\
\hline$H$ & $\begin{array}{l}\text { fog condition function, see } \\
\text { equation }(8)\end{array}$ \\
\hline I & $\begin{array}{l}\text { condition function for multiple } \\
\text { saturated regions, see equation (14) }\end{array}$ \\
\hline$K$ & $\begin{array}{l}\text { fog formation per unit volume } \\
{\left[\mathrm{kg} \mathrm{m}^{-3} \mathrm{~s}^{-1}\right]}\end{array}$ \\
\hline $\begin{array}{ll}L e_{\mathrm{v}} \\
t\end{array}$ & $\begin{array}{l}\text { modified Lewis number, } k / \rho c_{\mathrm{p}, \mathrm{v}} \mathbb{D} \\
\text { temperature }\left[{ }^{\circ} \mathrm{C}\right]\end{array}$ \\
\hline$y$ & $\begin{array}{l}\text { coordinate normal to the interface (or wall) } \\
{[\mathrm{m}] \text {. }}\end{array}$ \\
\hline
\end{tabular}
Greek symbol
$\delta \quad$ film thickness [m].
Subscripts
a boundary of saturated and superheated region
a, 1 boundary of saturated and intermediate superheated region
a,2 boundary of saturated and intermediate superheated region
b bulk
c diffusional
i interface, wall
$K=0$ pertaining to zero fog formation per unit volume
t thermal.

of multiple regions (in the next section), but it offers the advantage that the computational effort is reduced (see refs. $[1,2])$.

At the boundary of the saturated and superheated regions the vapour fraction and temperature (and physical properties) are continuous, as are the energy and mass flux. This results in

$$
F\left(t_{\mathrm{a}}\right)=G\left(t_{\mathrm{a}}\right)\left(\equiv c_{\mathrm{a}}\right)
$$

and

$$
\left.\frac{\mathrm{d} F}{\mathrm{~d} t}\right|_{t_{\mathrm{a}}}=\left.\frac{\mathrm{d} G}{\mathrm{~d} t}\right|_{t_{\mathrm{a}}}\left(\equiv L e_{\mathrm{v}} \frac{c_{\mathrm{a}}-1}{t_{\mathrm{b}}-t_{\mathrm{a}}}\left(\exp \left(\frac{1}{L e_{\mathrm{v}}} \ln \left(\frac{1-c_{\mathrm{b}}}{1-c_{\mathrm{a}}}\right)\right)-1\right)\right) .
$$

Equations (5) and (6) prescribe the gradient-continuity of $c$ and $t$ in the film. As long as the left-hand side of equation (6) is larger than the right-hand side, the sought $t_{\mathrm{a}}$ will be larger. In this way the $t_{\mathrm{u}}$ (and $c_{\mathrm{a}}=F\left(t_{\mathrm{a}}\right)$ ) can be determined iteratively. Additional information about the use and the features of the slope condition (2) and the tangency condition (6) is given in ref. [1].

In refs. $[1,2]$ it was furthermore demonstrated that in the saturated region the fog formation per unit volume reads

$$
K=\rho \mathbb{D} \frac{\left(L e_{\mathrm{v}}-1\right)\left(\frac{1}{1-F} \frac{\mathrm{d} F}{\mathrm{~d} t}\right)^{2}+L e_{\mathrm{v}} \frac{1}{\mathrm{I}-F} \frac{\mathrm{d}^{2} F}{\mathrm{~d} t^{2}}}{L e_{\mathrm{v}}+\frac{H_{\mathrm{lat}}}{c_{\mathrm{p}, \mathrm{v}}} \frac{\mathrm{I}}{\mathrm{I}-F} \frac{\mathrm{d} F}{\mathrm{~d} t}}\left(\frac{\mathrm{d} t}{\mathrm{~d} y}\right)^{2} .
$$

This expression follows from combining the energy and diffusion equation in the fogging region. For $L e_{v}<1$ it is conceivable that $K$ becomes zero or even negative, thus fog formation ends. Mathematically fog formation in the film ends when the numerator of equation (7) becomes zero (or negative)

$$
L e_{\mathrm{v}} \leqslant H(t)=\frac{\left(\frac{\mathrm{d} F}{\mathrm{~d} t}\right)^{2}}{\left(\frac{\mathrm{d} F}{\mathrm{~d} t}\right)^{2}+(1-F) \frac{\mathrm{d}^{2} F}{\mathrm{~d} t^{2}}}
$$

where the fog condition function $H(t)$ has been introduced. In refs. $[1,2]$ it has been demonstrated that $0<F(t) \leqslant 1$ and $0<H(t) \leqslant 1$, and that both $F(t)$ and $H(t)$ (and their first derivatives with respect to $t$ ) are monotonically increasing functions in $t$. Furthermore, for a mixture of air and water vapour at atmospheric pressure $F(t)$ and $H(t)$ have actually been computed and depicted.

As said, for $L e_{\mathrm{v}}<1$ it is possible that $K=0$, the pertaining temperature being denoted by $t_{K=0}$ (thus $\left.L e_{\mathrm{v}}=H\left(t_{K-0}\right)\right)$. In
Appendix B of ref. [1] it was assessed that the saturated region never attains this temperature; $t_{K=0}>t_{\mathrm{a}}$. Hence $K>0$ is guaranteed in the saturated part of the film (sce Fig. 2). Moreover, this insight was sustained by some performed computations concerning wall-condensing water vapour in air.

The main features of the fog film model have been summarized. So far, the analysis has excluded the presence of multiple superheated and saturated regions in the film. However, in Appendix B of ref. [1] it was explained that this could be possible for $L e_{\mathrm{v}}<1$ as $G(t)$ is then a convex curve (likewise $F(t))$. In the next section such multiple regions are examined.

\section{MULTIPLE SUPERHEATED AND SATURATED REGIONS}

Assume there is a second superheated region, $\delta_{\mathrm{a}, 1} \leqslant y \leqslant$ $\delta_{\mathrm{a}, 2}$, situated in the saturated region $0 \leqslant y \leqslant \delta_{\mathrm{a}}$, whereby $0 \leqslant \delta_{\mathrm{a}, 1} \leqslant \delta_{\mathrm{a}, 2} \leqslant \delta_{\mathrm{a}}$ (see Fig. 2). At the boundaries of this intermediate superheated region, $t$ attains the values $t_{\mathrm{a}, 1}$ and $t_{\mathrm{a}, 2}$, and $c$ the values $c_{\mathrm{a}, 1}$ and $c_{\mathrm{a}, 2}$. In this superheated region $t(y)$ and $c(y)$ can be determined and $y$ eliminated, yielding as a relation in this region

$c=G(t)$

$$
\begin{array}{r}
=1+\left(c_{\mathrm{a}, 1}-1\right)\left(\frac{t-t_{\mathrm{a} .1}}{t_{\mathrm{a} .2}-t_{\mathrm{a}, 1}}\left(\exp \left(\frac{1}{L e_{\mathrm{v}}} \ln \left(\frac{1-c_{\mathrm{a} .2}}{1-c_{\mathrm{a}, 1}}\right)\right)-1\right)+1\right)^{L u_{\mathrm{v}}} \\
\left(t_{\mathrm{a}, 1} \leqslant t \leqslant t_{\mathrm{a} .2}\right) . \quad(9)
\end{array}
$$

Both at $y=\delta_{a, 1}$ and $y=\delta_{a, 2}$ the gradient-continuity conditions (5) and (6) have to be fulfilled. Applying equations (5), (6) and (9) at $y=\delta_{2,1}$ yields

$$
\begin{aligned}
\left.\frac{\mathrm{d} G}{\mathrm{~d} t}\right|_{t, \mathrm{a}, \mathrm{t}} & =L e_{\mathrm{v}}\left(\frac{F\left(t_{\mathrm{a}, 1}\right)-1}{t_{\mathrm{a}, 2}-t_{\mathrm{a} .1}}\right)\left(\exp \left(\frac{1}{L e_{\mathrm{v}}} \ln \left(\frac{1-F\left(t_{\mathrm{a}, 2}\right)}{1-F\left(t_{\mathrm{a}, 1}\right)}\right)\right)-1\right) \\
& =\left.\frac{\mathrm{d} F}{\mathrm{~d} t}\right|_{t_{\mathrm{t}, 1}} .
\end{aligned}
$$

At $y=\delta_{\mathrm{a} .2}$ these equations yield

$$
\begin{aligned}
\left.\frac{\mathrm{d} G}{\mathrm{~d} t}\right|_{t_{\mathrm{a}, 2}}= & L e_{\mathrm{v}} \exp \left(\frac{L e_{\mathrm{v}}-1}{L e_{\mathrm{v}}} \ln \left(\frac{1-F\left(t_{\mathrm{a}, 2}\right)}{1-F\left(t_{\mathrm{a}, 1}\right)}\right)\right)\left(\frac{F\left(t_{\mathrm{a}, 1}\right)-1}{t_{\mathrm{a}, 2}-t_{\mathrm{a}, 1}}\right) \\
& \times\left(\exp \left(\frac{1}{L e_{\mathrm{v}}} \ln \left(\frac{1-F\left(t_{\mathrm{a}, 2}\right)}{1-F\left(t_{\mathrm{a}, 1}\right)}\right)\right)-1\right)=\left.\frac{\mathrm{d} F}{\mathrm{~d} t}\right|_{t_{\mathrm{a}, 2}}
\end{aligned}
$$

Combining equations (10) and (11) yields the following condition :

$$
\left.\frac{\mathrm{d} F}{\mathrm{~d} t}\right|_{\mathrm{f}_{\mathrm{a}, 2}}=\left.\exp \left(\frac{L e_{\mathrm{v}}-1}{L e_{\mathrm{v}}} \ln \left(\frac{1-F\left(t_{\mathrm{a}, 2}\right)}{1-F\left(t_{\mathrm{a}, \mathrm{t}}\right)}\right)\right) \frac{\mathrm{d} F}{\mathrm{~d} t}\right|_{t_{\mathrm{a}, \mathrm{l}}}
$$




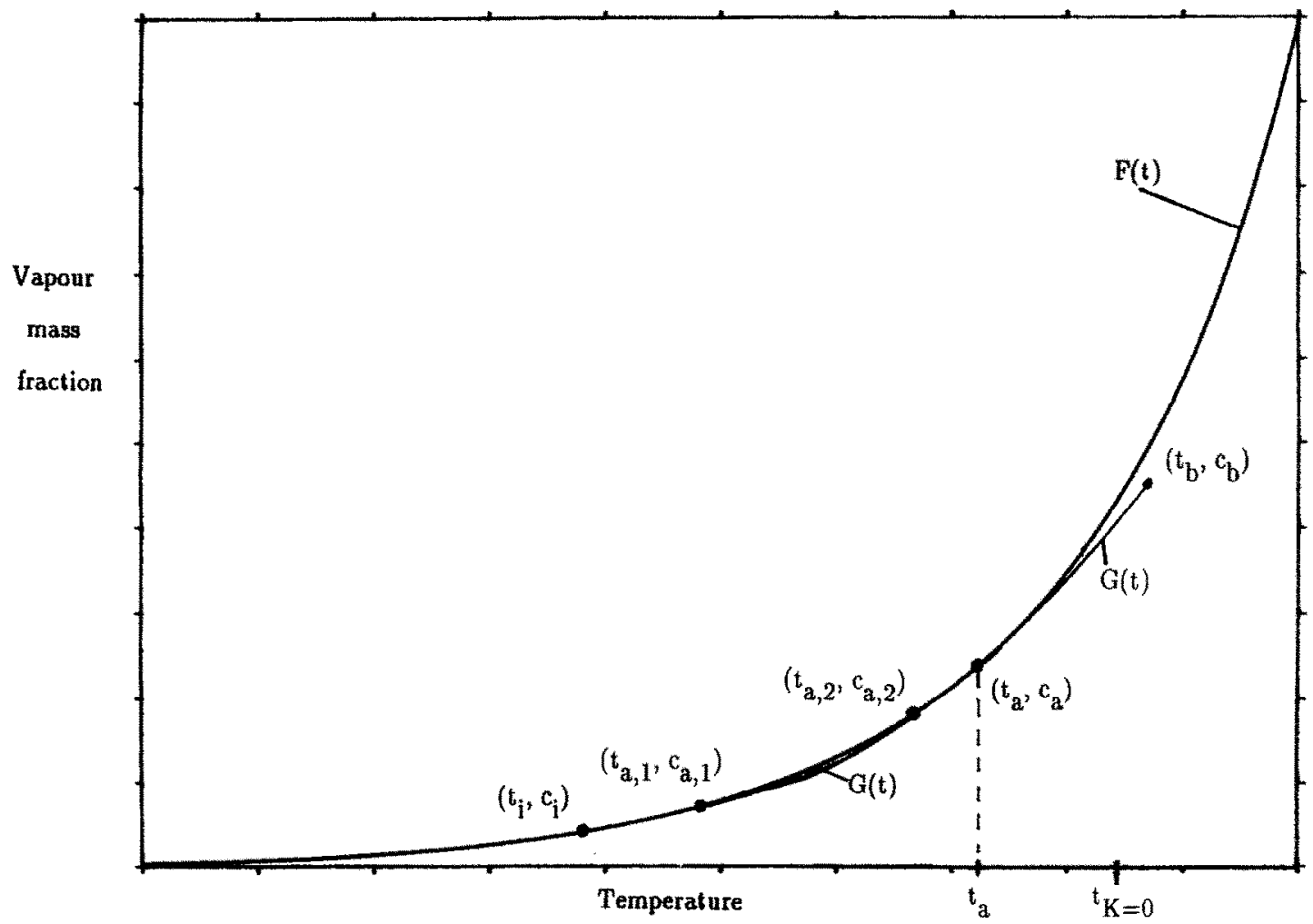

FIG. 2. Relation between $c$ and $t$ in the film ( $y$ eliminated), $L e_{v}<1, \delta_{t}=\delta_{\mathrm{t}}$ and condensation conditions.

This equation is rewritten as the following relation among $t_{\mathrm{i}, 1}, t_{\mathrm{i}, 2}$ and $L e_{\mathrm{v}}$

$$
I\left(t_{\mathrm{it} 1.1}, t_{\mathrm{an} .2}\right)=L e_{\mathrm{v}}
$$

where $I\left(t_{1,1}, t_{3,2}\right)$ follows from equations (12) and (13)

$$
I\left(t_{\mathrm{a}, 1}, t_{\mathrm{a}, 2}\right)=\frac{\ln \left(\frac{1-F\left(t_{\mathrm{a}, 1}\right)}{1-F\left(t_{\mathrm{a}, 2}\right)}\right)}{\ln \left(\frac{1-F\left(t_{\mathrm{a}, 1}\right)}{1-F\left(t_{\mathrm{a} .2}\right)}\right)+\ln \left(\left.\frac{\mathrm{d} F}{\mathrm{~d} t}\right|_{t, 2}\right)-\ln \left(\left.\frac{\mathrm{d} F}{\mathrm{~d} t}\right|_{\mathrm{a}, 1}\right)}
$$

It can readily be concluded that $I\left(t_{2,1}, t_{1,2}\right)<1$ since both $F(t)$ and $\mathrm{d} F / \mathrm{d} t$ are monotonically increasing functions in $t$ and $t_{\mathrm{a}, 2}>t_{\mathrm{a}, 1}$. This would be expected since an extra superheated region is possible only for convex $G(t)$, implying $L e_{v}$ $\left(=I\left(t_{\mathrm{a}, 1}, t_{\mathrm{a}, 2}\right)\right)<\mathrm{I} . I\left(t_{\mathrm{a}, 1}, t_{\mathrm{a}, 2}\right)$ is a monotonically decreasing function in both $t_{3,}$ and $t_{3,3}$, which is demonstrated in the following.

The lirst derivative of $I\left(t_{i, i}, t_{a, 2}\right)$ with respect to $t_{a, z}$ follows from differentiating equation (14) and substituting equation (12)

$$
\begin{aligned}
& \mathrm{d} l \quad \ln \left(\frac{1-F\left(t_{\mathrm{a}, \mathrm{l}}\right)}{1-F\left(t_{\mathrm{a}, 3}\right)}\right)
\end{aligned}
$$

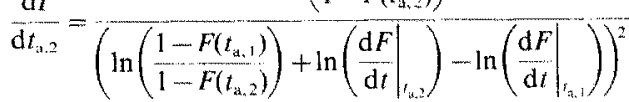

$$
\begin{aligned}
& -\left(L e_{\mathrm{v}}-1\right)\left(\left.\frac{\mathrm{d} F}{\mathrm{~d} t}\right|_{t_{\mathrm{x}}}\right)^{2}-\left.L e_{\mathrm{v}}\left(1-F\left(t_{3.2}\right)\right) \frac{\mathrm{d}^{2} F}{\mathrm{~d} t^{2}}\right|_{t_{2}} \\
& \left.L e_{v}\left(1-F\left(t_{\alpha, 2}\right)\right) \frac{\mathrm{d} F}{\mathrm{~d} t}\right|_{t_{\mathrm{s}, 2}}
\end{aligned}
$$

The sign of this first derivative is governed by the numerator of the second factor since the first factor and denominator of the second factor are both definitely positive. Reminding ourselves that $K>0$ (or $L e_{3}>H(t)$ for $t_{i} \leqslant t \leqslant t_{3}$, one can see that this numerator is smaller than zero (sce also equation (7)). Hence

$$
\mathrm{d} t_{\mathrm{iL}} \geqslant<0
$$

An analogous consideration of the first derivative of $f\left(t_{2,1}, t_{3,2}\right)$ with respect to $t_{3,-1}$ reveals

$$
\frac{\mathrm{d} I}{\mathrm{~d} t_{\mathrm{a} .1}}<0 \text {. }
$$

This can also be explained with equation (14); this function remains the same when $t_{i, 1}$ and $t_{3,2}$ are exchanged. Equations (16) and (17) imply that $I\left(t_{2,1}, t_{x, 2}\right)$ increases with decreasing $t_{1,}$ and $t_{3,}$. Consequently, $/(t, 1, t, 2)$ is greatest when both $t_{\mathrm{a}, 1}$ and $t_{\mathrm{i}, 2}$ attain their minimum value, that is $t_{1}$.

This maximum magnitude follows from equation (14), I'Hôpital's rule (as both the numerator and denominator tend to zero) and cquation (8)

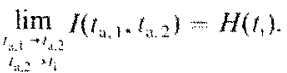

We have already discussed that for the considered region $t_{i} \leqslant t \leqslant t_{d}$, it holds that $H(t)<L e_{v}$. The fact that $I\left(t_{x, 1}, t_{a, 2}\right) \leqslant$ $H\left(t_{i}\right)$ implies that $I\left(t_{1,1}, t_{2,2}\right)<L e_{v}$ for $t_{i} \leqslant t_{i, 1} \leqslant t_{3}, \leqslant t_{i \cdot}$. In other words, there exist no $t_{a}$ and $t_{a}$, that can satisfy equation (13) and enable another superheated region in the film. Accordingly, multiple superheated and saturated regions are indeed not possible.

In this section only condensation eases have been discussed. For evaporation, or $t_{\mathrm{i}} \geqslant t_{\mathrm{a}, 1} \geqslant t_{\mathrm{a}, 2} \geqslant t_{\mathrm{a}} \geqslant t_{\mathrm{b}}$, it can be derived that $I\left(t_{\left.\mathrm{a}, 1+t_{\mathrm{at} 2}\right)}\right)$ is a monotonically increasing function in both $t_{\mathrm{a}, 1}$ and $t_{\mathrm{a}, 2}$. Consequently, the maximum $f\left(t_{3,1}, t_{1,2}\right)$ is again found at $t_{i, 1}=t_{3,2}=t_{k}$, resulting in 
expression (18). The same conclusion as for the condensation analysis can therefore be drawn with regard to multiple regions in the film.

\section{CONCLUSION}

In the present study the possible existence of multiple superhcatcd and saturated regions in a stagnant binary film, consisting of a vapour and non-condensables, has been examined. The analysis yields the important conclusion that, once fog formation has been detected with the slope condition and a saturated and superheated region determined with the tangency condition, the uniqueness of both regions is assured.
Acknowledgements - The author wishes to thank the management of Akzo Research Laboratories Arnhem for their permission to publish this paper and Messrs. H. P. Korstanje and $G$. Vegt for their support of this work.

\section{REFERENCES}

1. II. J. H. Brouwers, Film models for transport phenomena with fog formation, with application to plastic heat exchangers and condensers, Ph.D. Thesis, Eindhoven University of Technology (1990).

2. H. J. H. Brouwers, Film models for transport phenomena with fog formation : the fog film model, Int. J. Heat Mass Transfer 35, 13-28 (1992).

\section{The film model applied to free convection over a vertical plate with blowing or suction}

\section{H. J. H. Brouwers'}

Akzo Research Laboratories Arnhem, Fibers Division, Department of Mechanical Engineering, Velperweg 76, 6824 BM Arnhem, The Netherlands

(Received 27 May 1991 and in final form 19 July 1991)

\section{INTRODUCTION}

FOR MANY years now classical film model correction factors have been successfully used to predict the effect of mass transfer lowards a wall on transport phenomena, such as exerted friction and heat and mass transferred. The correction factors can be derived from a stagnant film analysis and applied to systems using either an imposed (transpiration) mass flux or a diffusional vapour flux (by condensation or evaporation). Recent reviews of the film model are found in Bannwart [1], Bannwart and Bontemps [2], Brouwers and Chesters [3] and Brouwers [4]. Whereas the former two authors extended the model to include the effect of mass transfer on film thickness, the latter investigators added fog formation to the model.

The film model expressions have been applied to forced convective heat flow in the presence of an imposed mass flux by Mickley ot al. [5] and Wang and Tu [6]. Colburn and Drew [7] and Webb and Sardesai [8], among others, fruitfully applied the film model to forced convective diffusional mass transfer. Heat transfer of forced pure vapour flow with wall condensation has been treated with the film model by Mizushina et al. [9]

With respect to free convective flow with mass transfer, the film model has been utilized only by Corradini [10] and Vernier and Solignac [11] in an attempt to model the postulated loss-of-coolant accident in a nuclear reactor. Turbulent free convective flow of wall condensing water vapour in air was considered, but poor agreement was found with the experiments performed. Until now, however, the film model predictions have never been applied to free convective flow problems with imposed wall transpiration. For example, fluid injection is an effective way of cooling and reducing heat transfer to surfaces in extremely hot surroundings.

Hence, in this technical note the classical film model is applied to free convective heat transfer with an imposed mass flux. Subsequently, the predictions are compared with the theoretical results of previous investigators. These results are based on an analysis of the governing equations of laminar

$\dagger$ Present address: Department of Civil Engineering, Twente University, P.O. Box 217, 7500 AE Enschede, The Netherlands. free convective boundary layer flow over a permeable vertical plate with wall transpiration. In this paper the comparison is restricted to laminar free convection because, to the author's knowledge, data on turbulent free convective flow with wall suction or injection are not yet available.

\section{FILM MODEL}

According to film theory the actual local Nusselt number, denoted by $N u_{x}$, in the presence of mass transfer follows from multiplying the zero suction (or neutral) Nusselt number, $N u_{x}^{*}$, by a correction factor

$$
N u_{x}=\Theta_{\mathrm{t} . \mathrm{flim}} N u_{x}^{*} .
$$

The thermal correction factor, commonly referred to as Ackermann correction, follows from Brouwers [4]-among others-as

$$
\Theta_{\mathrm{t}, \mathrm{film}}=\frac{-\phi_{1}}{\mathrm{e}^{-\phi_{1}}-1}
$$

where the dimensionless mass flux towards the wall reads

$$
\phi_{\mathrm{t}}=\frac{\dot{m} c_{\mathrm{p}}}{h_{x}^{*}}=-\frac{v_{\mathrm{w}} \rho c_{\mathrm{p}}}{h_{x}^{*}} .
$$

In this equation $h_{x}^{*}$ represents the local heat transfer coefficient in the case of zero mass transfer. For free convective heat transfer it is defined as

$$
h_{x}^{*}=\frac{N u_{x}^{*} k}{x}
$$

where $x$ is a coordinate along the plate. For free convection over an isothermal impermeable vertical plate by thermal buoyancy the local neutral Nusselt number (see Ostrach [12]) reads

$$
N u_{x}^{*}=\psi(\operatorname{Pr})\left(\frac{G r_{x}}{4}\right)^{1 / 4}
$$

In Table 1 values of $\psi$ are listed for various Prandtl numbers, taken from ref. [12]. In the next section the film model predictions are extensively compared with the results of previous investigators. 